\title{
Research on the education reform of TRX suspension training method applied in physical fitness course in Police Colleges
}

\author{
Zhang Fan ${ }^{1,2, a}$ \\ ${ }^{1}$ Department of Police Skills and Tactics, Nanjing Forest Police College, Nanjing 210023, China \\ ${ }^{2}$ Sports Science postdoctoral programme, Nanjing Normal University, Nanjing 210023, China \\ a zhangfan@nfpc.edu.cn
}

Keywords: TRX, suspension training, core muscles, Police Colleges, Police physical fitness.

\begin{abstract}
Total Body Resistance Exercise (TRX) suspension training system has been a popular method for athletes to improve their sports performance and enthusiasts. There are different training needs of groups began to try to use TRX as the training equipment, such as athletes, forces, fitness trainer, physical therapist, but because TRX is in recent years developed products, so TRX has been the theoretical background of the basic training, only a single pure and simple operation concept. Although many studies have pointed out that suspension training can strengthen the core muscle and increase performance, the study of TRX suspension equipment used in police physical training is rarely involved. Therefore, this study intends to use TRX as a training tool to design the corresponding training methods, in order to provide scientific reference for the reform and practice of the teaching content of physical fitness training courses for Police College students.
\end{abstract}

\section{Introduction}

With the acceleration of social and economic development in China, violent crime is on the increase. As an important role in maintaining social harmony, police should intensify their efforts to crack down on criminals [1]. And the basic condition of fighting criminals is to have a strong body and good Physical Fitness. However, it is influenced by the traditional concept of police physical training. The physical quality training of police college students still often follows the traditional model (in addition to learn cultural knowledge is the basic physical training). Paradoxically, the training methods and means of physical fitness on the slightly outdated, while running, push ups, and other traditional physical training mode of single parallel bars to the school students' physical quality promotion has a certain positive role, but compared with the recent update training concept. These traditional physical training methods may not play a substantive role, and even increase the chances of sports injuries and training benefits reduced. If the introduction of current physical training in the TRX training, serve the core muscle training (basic police skills and tactics ability of efficient execution), may be a useful attempt and the of effective supplement traditional physical training mode in Police Colleges.

\section{Theoretical basis of TRX suspension training}

TRX is a unique suspension training system, it can make the body sagittal and frontal plane and horizontal plane at the same time in use, and through a fixed anchor with one hand, hands, foot or feet hanging body end in contact with the ground relative, using its own weight and ground vertical angle as according to the adjustment of training intensity;

The reason why TRX is gradually accepted by the public comes from its characteristics and advantages (Table 1). TRX suspension training system is not only used to promote the physical fitness of healthy people, but also to promote the performance of athletes [2]. In theory, it can also be extended to the training of police physical fitness. 
Table 1 TRX suspension training advantage characteristics.

\begin{tabular}{|c|c|}
\hline Cost effectiveness & The price is low and the equipment is small in size. \\
\hline Space restriction & Indoor and outdoor, free from site restrictions. \\
\hline Operation difficulty & One person can operate. \\
\hline Training methods & Diversified and multi-faceted training model. \\
\hline $\begin{array}{c}\text { Training } \\
\text { characteristics }\end{array}$ & It can train more people at the same time and have more training efficiency. \\
\hline
\end{tabular}

\subsection{Theoretical basis of TRX suspension training}

The principle of TRX suspension training is the body of some or all of the above, suspended from the fixed point of the rope or sling, or removed by adjusting the effect of special exercise treatment techniques of gravity and friction action factors, including technical suspension neck, upper and lower limbs and trunk [3]. TRX suspension training aims at restoring proprioceptive control, motor control, increased core stability, muscle strength, endurance, and cardiovascular function to improve the overall functioning of the motor system.

\subsubsection{Relax muscles}

Through the support of the suspension system, the individual can do more stretching and supporting at the same time, and then help to relax and extend the muscles.

\subsubsection{Movement of joint motion}

The TRX suspension training to help them reduce the individual affected by gravity, reducing action difficult, also can increase the activity of joint training to master slowly, step by step, a gradual increase in the difficulty of training.

\subsubsection{Stable muscle training}

The deep core muscle refers to the muscles that are responsible for stabilizing the body, and the global muscles refers to the muscles that produce movement in the body. For example, the waist muscle is the most important for the stability of the deep abdominal muscle and multifidus muscle, when the body moves, the deep core muscles before systemic muscle contraction action to maintain stability [4]. TRX suspension training is mainly to train the stability of the deep muscles, focusing on the lower strength, isometric contraction of the deep stability of the muscles, which emphasizes the maintenance of time gradually increased, rather than increased weight.

\subsubsection{Proprioception training}

Proprioception training is an important element in the TRX suspension training concept, through the closed kinetic chain on the surface is not stable, in order to achieve the best stimulation, such as the frequent use of foam rubber cushion, balance board and inflatable rubber pad to increase instability, and thus stimulate the deep nuclear myocardial group.

\subsubsection{Closed power transmission chain training}

The locking power chain movement is mainly trained by the TRX suspension system. The load can be gradually changed by the arm arm, the different parts of the body, and the relative relationship between the body and the fulcrum so as to increase the intensity of movement [5]. Closed power transmission chain training allows deeper, stabilizing muscles and more coordinated movements of systemic muscles.

Above all, we understand that TRX suspension training can improve the strength and strength of the core muscles. The inference about the students, TRX can enhance the trunk muscle strength, the lumbar spine has good fixation, because the core muscles strong, effectively fixing spine, limbs and strengthen links, enhance physical fitness can therefore be better, in order to lay the cornerstone of police law enforcement ability. 


\section{Design of TRX suspension system training course}

The course was designed for twelve weeks of training, 90 minutes per lesson, and two lessons per week. The 90 minute training course is divided into three parts.

\subsection{Warm-up exercises}

The time is about 15 minutes. Low intensity aerobic exercise improves body temperature, and through the dynamic tensile ductility, increase muscle flexibility and increased ROM, sensory and motor neurons in series as the main training courses and to avoid sports injury.

\subsection{TRX suspension training}

\subsubsection{Training arrangement}

Time is 60 minutes. This study takes 10 body muscle muscle training as the main part of TRX suspension training, including pectoralis major muscle, trapezius, biceps, triceps brachii muscle, latissimus dorsi muscle, rectus abdominis, erector spinae, oblique muscle, femoral head four and unit two biceps. The principles of TRX suspension training are shown in table 2 .

Table 2 Principles of TRX suspension training.

\begin{tabular}{|c|c|}
\hline Before the course & Measure the maximum muscle strength (1RM). \\
\hline Repetitions & The number of repetitions is between 8 and 12. \\
\hline Repeated groups & Repeated groups in the first 6 weeks was 2, and the remaining 6 weeks for the 5. \\
\hline Weight & Adjust the load strength by angle. \\
\hline
\end{tabular}

\subsubsection{The muscular part of training}

Biceps training. Repeat 8 12RM to adjust the weight, body facing the anchor (TRX tie hanging point), with elbow flexion, biceps contraction as the main action, use body weight as weight, body and floor vertical load is lighter, heavier weight body and parallel to the floor.

Triceps training. Repeat $8 \sim 12 \mathrm{RM}$ to adjust the weight, the body back to the anchor (TRX tie hanging place), with elbow joint stretching, triceps contraction is the main action, use body weight as weight, body and floor vertical load is lighter, heavier weight body and parallel to the floor.

Ectopectoralis training. Repeat 8 12RM to adjust the weight, the body back to the anchor (TRX tie hanging place), with shoulder horizontal adduction, pectoralis major muscle contraction is the main action, use body weight as weight, body and floor vertical load is lighter, heavier weight body and parallel to the floor.

Dorsal muscle training. Repeat $8 \sim 12 \mathrm{RM}$ to adjust the weight, body facing the anchor (TRX tie hanging place), with shoulder abduction, dorsal muscle contraction level as the main action, use body weight as weight, body and floor vertical load is lighter, heavier weight body and parallel to the floor.

Latissimus dorsi training. Repeat $8 \sim 12 \mathrm{RM}$ to adjust the weight, the body in the anchor (TRX tie hanging Office) just below the frontal plane, to shoulder adduction, latissimus dorsi muscle contraction is the main action, use feet to support the weight of the auxiliary, both feet support weight is lighter, heavier weight feet off the ground.

Rectus abdominis training. Repeat $8 \sim 12 \mathrm{RM}$ to adjust the weight, body and feet supine in the right direction to the anchor (TRX tie hanging Office), with spinal flexion, abdominal muscle contraction is the main action body from the anchor far more light weight; the body supine and foot anchor (light load and reverse object instead), the body from the anchor far more heavy weight.

Erector spinae training. The number of repeated 8 12RM to adjust the weight, the body lying in the anchor (TRX tie hanging below the office) to the spine stretch, paraspinal muscle contraction is the main action, with both hands to surrender posture, the body from the anchor closer to load more light; hands stretched out from the far more heavy weight anchor.

Training of internal and external oblique muscles. Repeat the number 8 12RM to adjust the weight, the body facing the anchor (TRX tied crane), to the left and right of the spine, abdominal and external oblique contraction as the main action. Use the weight of the body as a load, the body and 
floor vertical load, the lighter the body parallel to the floor, the heavier the load.

Quadriceps femoris training. With repeated repetitions of 8 12RM to adjust weight, the body faces the anchor point (TRX sling), with the hip and knee flexion, and the contraction of the four femoral head as the main movement. The use of body weight and arm strength as a load, feet and ground, arm force, auxiliary weight, light weight, one foot off the ground, arm light grip, auxiliary weight heavier.

Musculi biceps femoris training. To repeat the number of $8 \sim 12 \mathrm{RM}$ to adjust weight-bearing, the body supine, and the feet hung on the anchor (TRX tied crane) below, in order to knee flexion, the contraction of the two biceps femoris as the main action. The use of the body as weight-bearing, hip flexion, the foot hook, light weight, hip joint stability, while the feet inside the hook weight.

\subsection{Relaxation exercises}

Time is 15 minutes.The use of low intensity aerobic or stretching action to do active recovery, accelerated training after the metabolism of waste discharge.After the training, tight muscles can be relieved, lactic acid accumulation is reduced, and muscles of each training site are stretched to reduce muscle soreness and fatigue after exercise.

\section{Conclusion}

The TRX suspension training program designed in this study aims to promote the development of the core muscle through enhancing abdominal muscle strength, abdominal muscle, isometric muscle endurance, back muscle strength, back muscle isometric muscle endurance. With a strong core muscle, it can improve the stability of the body, maintain the accuracy of body posture, increase exercise ability, enhance the dynamic and static balance ability, and reduce the incidence of musculoskeletal diseases. Obviously, the TRX suspension training to strengthen the core muscles, is of considerable importance for performing complex tasks of Policing Tactics of police college students in the future, that is the significance and value of this research.

\section{Acknowledgements}

This work was supported in part by the Project of the Fundamental Research Funds for the Central Universities under Grant LGZD201709, in part by the Project of China Postdoctoral Science Foundation under Grant 2017M611849, in part by Jiangsu Qing LAN Project under Grant 2017, and in part by Nanjing Forest police College Teaching Reform Project under Grant ZD17001 \& YB17001.

\section{References}

[1] Shao, A., YE, A.P. (2012)An empirical study of physical training model in Police Colleges. Journal of Hebei police Career Academy, 4, 73-75. (In Chinese)

[2] Gaedtke, A., Morat, T . (2015)TRX Suspension Training: A New Functional Training Approach for Older Adults - Development, Training Control and Feasibility. International Journal of Exercise Science,3, 224-233.

[3] Gulmez, I. (2016)Effects of Angle Variations in Suspension Push-Up Exercise. Journal of Strength \& Conditioning Research, 31, 1017-1023.

[4] Tinto, A., Campanella, M., Fasano, M. (2017)Core strengthening and synchronized swimming: TRX $^{\circledR}$ suspension training in young female athletes. J Sports Med Phys Fitness, 6, 744-751.

[5] Li, C. L. (2012) Suspension training for physical fitness training. China Sports Coaches, 4, 37-41. (In Chinese)

[6] Yu, K., Suk, M., Kang, S. (2015)Effects of Combined Resistance Training with TRX on Physical Fitness and Competition Times in Fin Swimmers . International Journal of Sport Studies, 5, 508-515. 